\section{(4) Check for \\ Assessment of the Left Ventricular Deformable Indices (Strain Components) in Different Echocardiography Systems} updates

\author{
Mersedeh Karvandi ${ }^{1}{ }^{*}$
}

${ }^{1}$ MD, Research Institute: Taleghani Hospital, Shahid Beheshti University of Medical Sciences, Tehran, Iran

${ }^{*}$ Corresponding author: Mersedeh Karvandi, MD, Research Institute: Taleghani Hospital, Shahid Beheshti University of Medical Sciences, Tehran, Iran. E-mail: DOI: 10.21859/ijcp-03041_mr.karvandi@sbmu.ac.ir

\begin{abstract}
This Article provides simplified, easy-to-understand descriptions of the echocardiographic software used in conjunction with different echocardiography machines, such as those from Toshiba, Philips, GE, and Siemens, and explains how these sophisticated systems can best be used to exploit fully their ability to deliver more precise diagnoses and assist in treatment choice and follow-up. A variety of applications are covered, with presentation of algorithms and highlighting of tips and tricks. It will be of value for cardiologists, other interested clinicians, those pursuing fellowships in echocardiography, and sonographers; it will also be highly relevant for biomedical engineers, bio-mathematicians, computer scientists, and researchers in medical physics. There would be enormous clinical benefits of any non-invasive technique to estimate the true level of wall abnormal motions. Strain and strain rate are deformation measures. If different components of an object have different velocities, the object shape may be changed. In this article, we mentioned the left ventricular 2dimensional strain in normal hearts for each different type of strain methods from 5 echocardiography machines and we have also introduced MATLAB software as an echocardiography desk..
\end{abstract}

\section{INTRODUCTION}

Mathematics has made contributions to many fields, such as physics, engineering, computer science, medicine and economics. If we could provide a methodology that could have the ability to accurately predict the magnitude of myocardial diseases similar to the exactitude of more invasive methods, then it would certainly say that we have a winner application. Using non-invasive (and less expensive) procedures to make such critical diagnoses would be of huge relevance. There would be enormous clinical benefits of any noninvasive method to estimate the true level of wall abnormal motions (like ischemic heart diseases), such as avoiding an invasive study like cardiac catheterization with all its risks, the ability to repeat it on demand (serial measurements every 6 months or 1 year) which cannot be done with a cardiac catheterization and expenses (probably the most important) for an outpatient would be extremely low.

The parameter "strain" can be calculate via altered mathematical techniques, for example tissue Doppler imaging (TDI) joint with velocity gradient (VG), Speckle tracking- end 7 points, speckle tracking (ST),
Speckle tracking collective with TDI, 2-dimentional (2D) base strain (Speckle tracking united with velocity vector imaging (VVI)) and left ventricular (LV) modeling as a polygon shape combined with Speckle tracking and even simple M-mode is able to quantity a special kind of strain. Therefore the value of strain be governed by on how one processes the strain and what thoughtful of strain is meant (Longitudinal, Radial, Circumferential strain etc.). The following review provides a general overview of the currently available imaging techniques that enable strain measurements based on the available literature and on-going works [13]. This manuscript also introduces a reference value for strain, in healthy subjects, which is worthwhile in future basic and clinical research by the 5 specified software packages and MATLAB environment.

Different mathematical algorithms to quantify LV 2D strains:

1- Tissue Doppler imaging algorithm based on ultrasound method combined with velocity gradient (TDI-VG) [4]: 
- Echocardiographic images are acquired from LV short axis and apical views.

- The region of interest $L_{1}$ in the left ventricular myocardium is manually tracked with velocity $V_{1}$

- After passing time t the search region $L_{2}$ is detected by speckle tracking software with velocity $V_{2}$ (Fig 1 ).

- Let $L$ be the distance between $L_{1}$ and $L_{2}$ then strain rate $\left(\varepsilon^{\prime}\right)$ of $L_{1}$ at time $\mathrm{t}$ is formulated using TDI-VG method by the following way:

$$
\varepsilon^{\prime}\left(L_{1}, t\right)=\frac{V_{2}(t)-V_{1}}{L(t)}
$$

- The strain magnitude of the region of interest $L_{1}$ at time $t$ is:

$$
\varepsilon\left(L_{1}, t\right)=\int_{0}^{t} \varepsilon^{\prime}\left(L_{1}, u\right) d u
$$

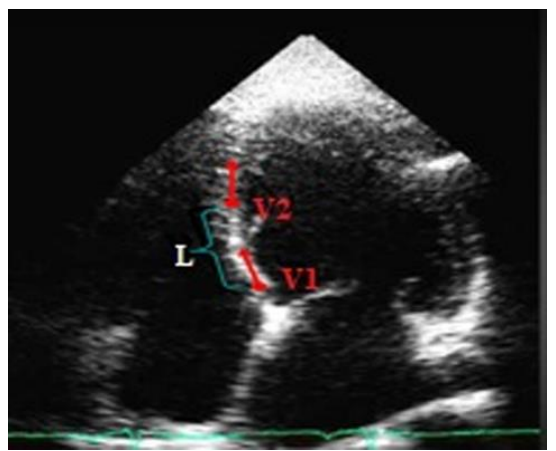

Figure 1: The TDI-VG based Method to obtain Strain by Time Integrating the Strain Rate Curve. $V_{1}$, Velocity at the Region of Interest $1 ; V_{2}$, Velocity at the Search Region 2; L, Distance between 1 and 2 Regions.
2- Speckle tracking combined with end-7 points algorithm (ST-end 7 points) [5-9]:

- Echocardiographic images are acquired from LV short axis and apical views.

- Seven known constant points are automatically traced in the left ventricular myocardium by speckle tracking software (Fig 2).

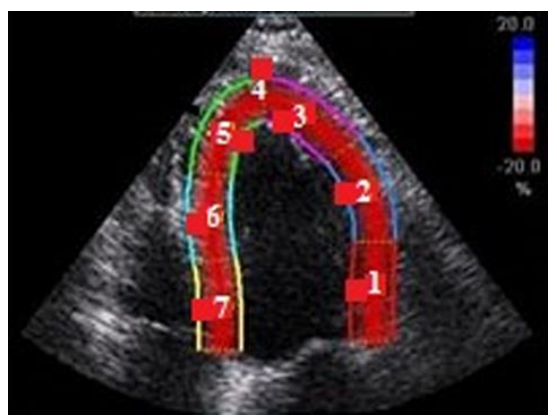

Figure 2: The ST-end 7 Points based Method to Obtain Strain by Displacement Tracking of 7 Points (1-7) per a Cardiac Cycle.

3- $j_{n}$; Search regions of the ith point at time $t_{n}$ in the nth frame (Fig 3).

- $L_{n, t_{n}}{ }^{\prime} s$; Distances between ith point and $j_{n}$ corresponding to time $t_{n}$.

- Strain value of the ith point at time $t_{n}$ :

$$
\varepsilon\left(i, t_{n}\right)=\sum_{k=1}^{n-1}\left(L_{k+1, t_{k+1}},-L_{k, t_{k}}\right) / L_{k, t_{k}}
$$
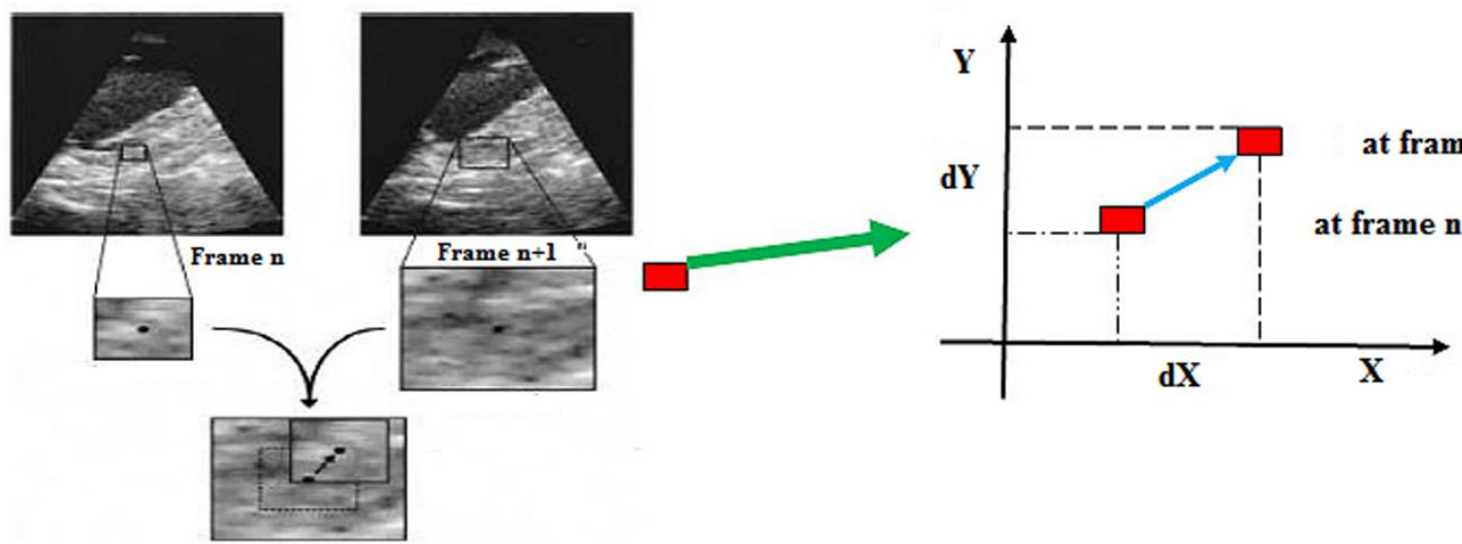

Figure 3: The Only Speckle Tracking Method to Obtain Strain by Searching the Region of Interest Frame by Frame through a Cardiac Cycle. dX, Speckle Displacement from Frame $n$ to Frame $n+1$ along X-Axis; dY, Speckle Displacement from Frame $n$ to Frame $n+1$ along Y-Axis.

4- Speckle tracking combined with TDI algorithm (Fig 4) $[10,11]$ :

- Echocardiographic images are acquired from LV short axis and apical views.

- Let $\mathrm{A}$ is an arbitrary region of interest which is traced manually.

- B to N, search points that are automatically tracked by the speckle tracking software.
- $L_{n}$, the change of length from point $\mathrm{A}$ to $\mathrm{N}$ in the $\mathrm{n}$-th frame.

- $T_{i}{ }^{\prime} s$, time corresponding to the search points.

- $L_{A N, n}$, the specified area corresponding to $\mathrm{N}$ at TDI velocity gradient diagram in the $\mathrm{n}$-th frame.

- Strain value of the point $\mathrm{A}$ at $\operatorname{tim} T_{i}$ is measurable by the following formula: 


$$
\begin{gathered}
\varepsilon\left(A, T_{i}\right)=\sum_{n=1}^{i}\left(L_{A N, n}\left(T_{i}\right)\right. \\
\left.-L_{A(N-1), n-1}\left(T_{i-1}\right)\right) \\
/ L_{A(N-1), n-1}\left(T_{i-1}\right)
\end{gathered}
$$

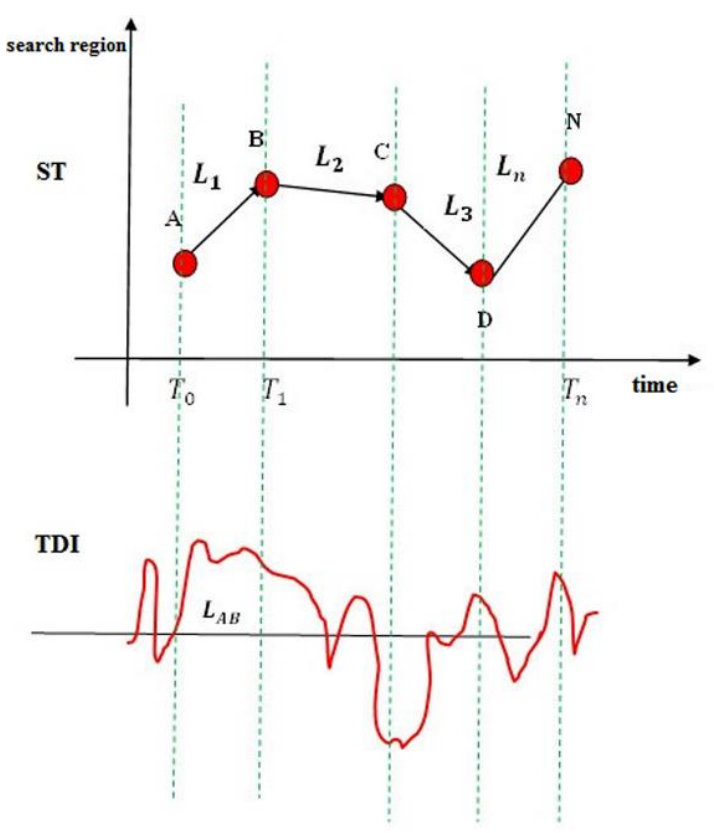

Figure 4: The ST-TDI based Method to obtain Strain by Combining Speckle Displacements to TDI Displacements. A, Region of Interest; B to N, Search Points; $T_{i}{ }^{\prime} s$, Time Corresponding to each Search Points; $L_{1}$ to $L_{n}$, the Changes of Lengths from Point A to N; $L_{A B}$, the Specified Area corresponding to $L_{1}$.

4- Velocity vector imaging algorithm (VVI) [12]:

- Echocardiographic images are acquired from LV 4 apical chamber (4C) views.

- $A_{1, k}$ 's; Regions of interests of the left ventricular myocardium that are manually divided to 13 points at 4C (1th frame); $k=1,2,3, . ., 13$ (Fig 5).

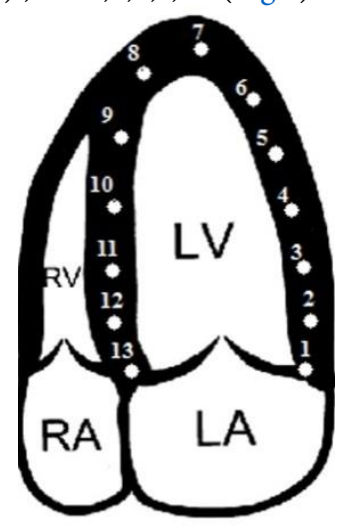

Figure 5: LV Myocardial Region has been divided to 13 Points at a 4C View.

5- Considering perpendicular lines crossing each divided points $(\mathrm{k}=1,2,3, \ldots, 13)$ (Fig 6)

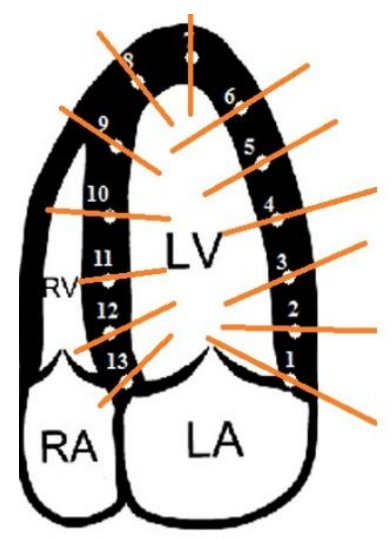

Figure 6: Perpendicular Lines crossing each 13 Points

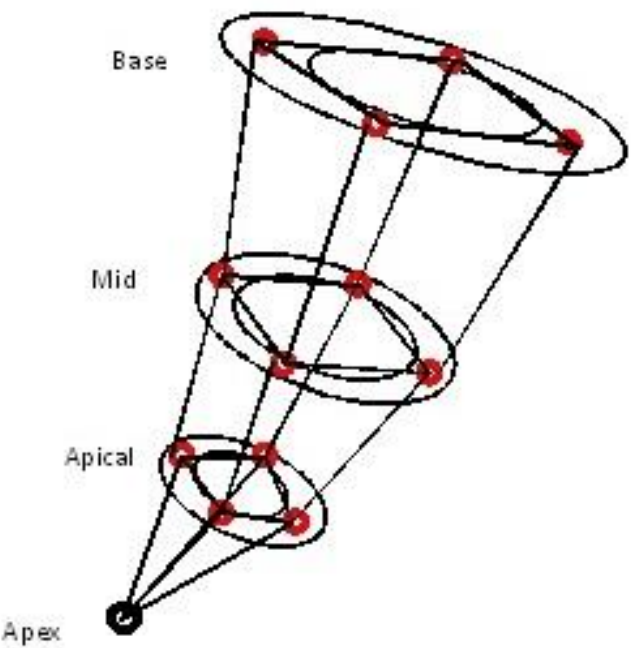

Figure 7: Red Points are corresponding Geometrical Points to Material Points of LV Myocardium. Material Points are usually selected as Contact Points in the Myocardium.

- $A_{2, k}$; New points are automatically identified from the intersection between perpendicular lines and the second frame $(\mathrm{k}=1,2,3 \ldots, 13)$

- $A_{i, k}$; New regions by the same procedures at last steps for the i-th frame (from 1-th frame to ith frame).

- $T_{i}$; Time corresponding to ith frame.

- $l_{i+1, T_{i}, k}$; Distances between $A_{i+1, k}$ and $A_{i, k}$ corresponding to $T_{i}$ for each $\mathrm{k}=1,2,3, \ldots, 13$.

$A 1, k, T i+1=j=1 j=i+1 l j+1, T j+1, k-l j, T j$,

$k l j, T j, k$-Strain value of points $A 1, k$ for each $\mathrm{k}$ at time $T_{i+1}$

5- Speckle tracking combined with $\mathrm{LV}$ as a polygon shape (a $4 D=3 D+1 D$ strain which its $3 \mathrm{D}$ is due to left ventricle as the polygon modeling and $1 \mathrm{D}$ is over the time) algorithm [13]:

- Echocardiographic images are acquired from LV short axis and apical views.

- The regions of left ventricular myocardium are divided 
to 7 points and 4 points at $4 \mathrm{C}$ and $\mathrm{SX}$ views respectively. - Myocardial point connections together can be realized by a polygon (Fig 7 ).
- Speckle tracking method applied to reconstructed LV as a polygon (Fig 8)

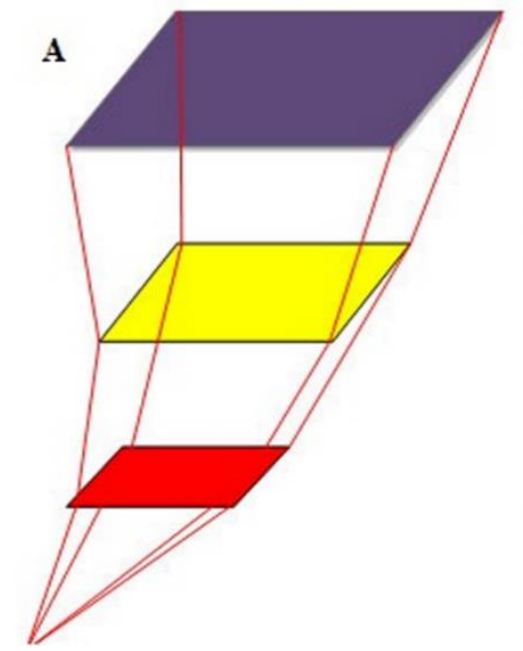

B

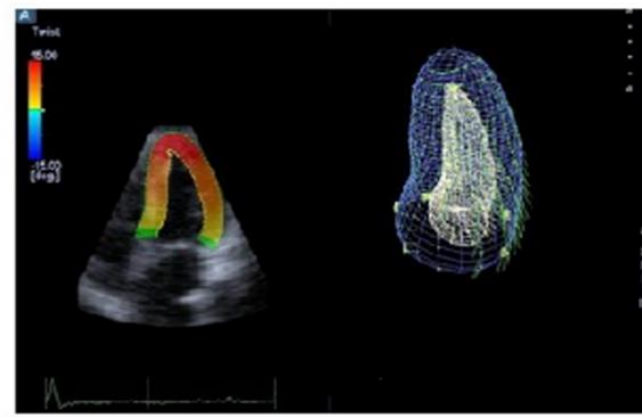

Figure 8: A) The Left Ventricle as a Polygon Shape Modeling. B) Speckle Tracking Method applied to LV Polygon Shape. LV, Left Ventricle.

Table 1: Left ventricular normal global strain components at 5 different modern echocardiography systems

\begin{tabular}{|lcccc|}
\hline & GLthod & GLL & GLCS & \\
E1 & TDI-VG & $-18.33 \pm 5.66 \%$ & $36.6 \pm 10.2 \%$ & $-21.5 \pm 5.7 \%$ \\
E2 & ST- end 7 Points & $-21.3 \pm 2.1 \%$ & $54.6 \pm 12.6 \%$ & $-22.8 \pm 2.9 \%$ \\
E3 & ST-TDI & $-18.9 \pm 2.5 \%$ & $36.3 \pm 8.2 \%$ & $-22.2 \pm 3.2 \%$ \\
E4 & ST-VVI & $-20.66 \pm 4.0 \%$ & $52.1 \pm 9.6 \%$ & $-31.11 \pm 5.8 \%$ \\
E5 & ST- LV as a polygon shape & $-19.9 \pm 2.4 \%$ & $51.4 \pm 8.0 \%$ & $-30.5 \pm 3.8 \%$ \\
\hline
\end{tabular}

Different Ultrasound Machines applied to Computations of Left Ventricular Global Strains

Different echocardiography systems can measure LV strain using above mathematical algorithms (1-5):

E1 = Vivid 7, GE Healthcare system (TDI-VG).

E2 = Vivid E9, GE Healthcare system (ST-end 7 points).

E3 $=$ iE33, Philips Medical system (ST-TDI).

E4 $=$ Siemens Medical system (ST-VVI).

E5 = Artida or Aplio, Toshiba Medical Systems (ST- LV as a polygon shape).

\section{MATLAB Software as an Echocardiography Desk}

One can also calculate the deformable indices by using MATLAB software offline in his or her personal computer. MATLAB is a sophisticated technical computing language and interactive environment for algorithm progress, data visualization, data analysis, and numeric computation. This software can easily be installed to your personal computer or laptop. MATLAB can be employed in a wide range of applications, including signal and image processing, test and measurement. It suffices to be imported echocardiographic images in MATLAB environment and to be used personally from related tools and commands into strain measurements.

\section{The Global Strain Values in a Normal Heart}

We have reviewed explicitly the left ventricular 2D strain in healthy subjects for each different type of strain methods from 5 echocardiography machines Table 1.

GLLS, global longitudinal strain; GLRS, global radial strain; GLCS, global circumferential strain; ST, speckle tracking; LV, left ventricle, TDI, tissue Doppler imaging; VG, velocity gradient; VVI, velocity vector imaging; E1, Vivid 7, GE Healthcare system; E2, Vivid E9, GE Healthcare system; E3, iE33, Philips Medical system; E4, Siemens Medical system; E5, Artida or Aplio, Toshiba Medical Systems.

\section{CONCLUSIONS}

To conclude, the emphasis is on the most recent advances in software and emerging benefits. In addition to its clinical relevance, the highlights relevant links between cardiology and the basic sciences and should assist in promoting future novel research that will further advance the field.

\section{Conflict of Interest}

There is no conflict of interest. 


\section{REFERENCES}

1. Geyer H, Caracciolo G, Abe H, Wilansky S, Carerj S, Gentile F, et al. Assessment of Myocardial Mechanics Using Speckle Tracking Echocardiography: Fundamentals and Clinical Applications. J Am Soc Echocardiogr. 2010;23(4):351-69; quiz 453-5. doi: 10.1016/j.echo.2010.02.015 pmid: 20362924

2. Mondillo S, Galderisi M, Mele D, Cameli M, Lomoriello VS, Zaca V, et al. Speckle-Tracking Echocardiography: a New Technique for Assessing Myocardial Function. J Ultrasound Med. 2011;30(1):71-83. pmid: 21193707

3. Voigt JU, Pedrizzetti G, Lysyansky P, Marwick TH, Houle H, Baumann R, et al. Definitions for a Common Standard for $2 \mathrm{~d}$ Speckle Tracking Echocardiography: Consensus Document of the Eacvi/Ase/Industry Task Force to Standardize Deformation Imaging. J Am Soc Echocardiogr. 2015;28(2):183-93. doi: 10.1016/j.echo.2014.11.003 pmid: 25623220

4. Heimdal A, Stoylen A, Torp H, Skjaerpe T. Real-Time Strain Rate Imaging of the Left Ventricle by Ultrasound. J Am Soc Echocardiogr. 1998;11(11):1013-9. pmid: 9812093

5. Amundsen BH, Crosby J, Steen PA, Torp H, Slordahl SA, Stoylen A. Regional Myocardial Long-Axis Strain and Strain Rate Measured by Different Tissue Doppler and Speckle Tracking Echocardiography Methods: a Comparison with Tagged Magnetic Resonance Imaging. Eur J Echocardiogr. 2009;10(2):229-37. doi: 10.1093/ejechocard/jen201 pmid: 18650220

6. Amundsen BH, Helle-Valle T, Edvardsen T, Torp H, Crosby J, Lyseggen E, et al. Noninvasive Myocardial Strain Measurement by Speckle Tracking Echocardiography: Validation Against Sonomicrometry and Tagged Magnetic Resonance Imaging. J Am Coll Cardiol. 2006;47(4):789-93. doi: 10.1016/j.jacc.2005.10.040 pmid: 16487846

7. Langeland S, D'Hooge J, Wouters PF, Leather HA, Claus P, Bijnens B, et al. Experimental Validation of a New Ultrasound Method for the Simultaneous Assessment of Radial and
Longitudinal Myocardial Deformation Independent of Insonation Angle. Circulation. 2005;112(14):2157-62. doi: 10.1161/CIRCULATIONAHA.105.554006 pmid: 16203928

8. Rappaport D, Adam D, Lysyansky P, Riesner S. Assessment of Myocardial Regional Strain and Strain Rate by Tissue Tracking in B-Mode Echocardiograms. Ultrasound Med Biol. 2006;32(8):1181-92.

10.1016/j.ultrasmedbio.2006.05.005 pmid: 16875953

9. Toyoda T, Baba H, Akasaka T, Akiyama M, Neishi Y, Tomita J, et al. Assessment of Regional Myocardial Strain by a Novel Automated Tracking System from Digital Image Files. J Am Soc Echocardiogr. 2004;17(12):1234-8. doi: 10.1016/j.echo.2004.07.010 pmid: 15562260

10. Cho GY, Chan J, Leano R, Strudwick M, Marwick TH. Comparison of Two-Dimensional Speckle and Tissue Velocity Based Strain and Validation with Harmonic Phase Magnetic Resonance Imaging. Am J Cardiol. 2006;97(11):1661-6. doi: 10.1016/j.amjcard.2005.12.063 pmid: 16728234

11. Hanekom L, Cho GY, Leano R, Jeffriess L, Marwick TH. Comparison of Two-Dimensional Speckle and Tissue Doppler Strain Measurement During Dobutamine Stress Echocardiography: an Angiographic Correlation. Eur Heart J. 2007;28(14):1765-72. doi: 10.1093/eurheartj/ehm188 pmid: 17573381

12. Mor-Avi V, Lang RM, Badano LP, Belohlavek M, Cardim NM, Derumeaux G, et al. Current and Evolving Echocardiographic Techniques for the Quantitative Evaluation of Cardiac Mechanics: Ase/Eae Consensus Statement on Methodology and Indications Endorsed by the Japanese Society of Echocardiography. Eur J Echocardiogr. 2011;12(3):167-205. doi: 10.1093/ejechocard/jer021 pmid: 21385887

13. Orderud F, Kiss G, Langeland S, Remme EW, Torp HG, Rabben SI. Combining Edge Detection with Speckle-Tracking for Cardiac Strain Assessment in 3d Echocardiography. Ultrasonics Symp. 2008:1959-62. doi: 10.1109/ultsym.2008.0483 